\title{
Diacronie
}

Studi di Storia Contemporanea

$N^{\circ} 35,3 \mid 2018$

Gli strumenti di Clio

\section{Le politiche abitative della Roma fascista}

L'esempio della Borgata Popolarissima di Tormarancia

\section{Flavio Conia}

\section{(2) OpenEdition}

Journals

\section{Edizione digitale}

URL: http://journals.openedition.org/diacronie/8925

DOI: 10.4000/diacronie.8925

ISSN: 2038-0925

Editore

Association culturelle Diacronie

Notizia bibliografica digitale

Flavio Conia, « Le politiche abitative della Roma fascista », Diacronie [Online], № 35, 3 | 2018, documento 4, Messo online il 29 septembre 2018, consultato il 01 mai 2019. URL : http:// journals.openedition.org/diacronie/8925; DOI : 10.4000/diacronie.8925 


\title{
Diacronie
}

Studi di Storia Contemporanea

$35,3 / 2018$

Gli strumenti di Clio: uomini, luoghi e teorie della storia dalla tradizione critica alla comunicazione digitale

\section{Le politiche abitative della Roma fascista: l'esempio della Borgata Popolarissima di Tormarancia}

\author{
Flavio CONIA
}

Per citare questo articolo:

CONIA, Flavio, «Le politiche abitative della Roma fascista: l'esempio della Borgata Popolarissima di Tormarancia», Diacronie. Studi di Storia Contemporanea : Gli strumenti di Clio: uomini, luoghi e teorie della storia dalla tradizione critica alla comunicazione digitale, 35, 3/2018, 29/09/2018,

URL: < http://www.studistorici.com/2018/09/29/conia_numero_35/ >

Diacronie Studi di Storia Contemporanea $\rightarrow$ http://www.diacronie.it

Rivista storica online. Uscita trimestrale.

redazione.diacronie@hotmail.it

Comitato di direzione: Naor Ben-Yehoyada - João Fábio Bertonha - Christopher Denis-Delacour - Maximiliano Fuentes Codera Anders Granås Kjøstvedt - John Paul Newman - Deborah Paci - Niccolò Pianciola - Spyridon Ploumidis - Wilko Graf Von Hardenberg

Comitato di redazione: Jacopo Bassi - Luca Bufarale - Gianluca Canè - Luca G. Manenti - Fausto Pietrancosta - Alessandro Salvador - Matteo Tomasoni - Luca Zuccolo Creative Commons 3.0. Possono essere riprodotti e modificati a patto di indicare eventuali modifiche dei contenuti, di riconoscere la paternità dell'opera e di condividerla allo stesso modo. La citazione di estratti è comunque sempre autorizzata, nei limiti previsti dalla legge. 


\title{
4/ Le politiche abitative della Roma fascista: l'esempio della Borgata Popolarissima di Tormarancia
}

\author{
Flavio CONIA
}

L'elaborato si pone come obiettivo la ricostruzione delle vicende che diedero vita ad una delle borgate popolarissime della Roma degli anni Trenta, Tormarancia. Le demolizioni dovute al nuovo piano regolatore fascista, il tentativo di rilancio dell'immagine di Roma attraverso vaste operazioni di decoro mirate ad abbattere insediamenti abusivi e baracche: l'emergenza abitativa diviene materia di complessa gestione ed una vera piaga per la Capitale. Grazie all'Archivio Storico Capitolino è possibile ricostruire le storie e le condizioni di vita degli "ultimi" che popolavano Roma: le schede personali di ogni nuovo inquilino della borgata risultano essere un vero e proprio "tesoro d'archivio", ricco di informazioni preziose per un'indagine socio-antropologica dei primi abitanti delle borgate romane.

\section{Verso la costruzione di Tormarancia: gli sbaraccamenti e gli sfratti a Roma. Un inquadramento storico}

«Ebbene, io vivo a Tormarancio, con mia moglie e sei figli, in una stanza che è tutta una distesa di materassi, e quando piove, l'acqua ci va e viene come sulle banchine di Ripetta» ${ }^{1}$ : così Alberto Moravia descriveva Tormarancia nel suo racconto Il Pupo del 1954, vent'anni dopo l'avvio dei lavori per la costruzione della borgata oggetto di studio di questo articolo. Una narrazione non romanzata, ma ben attinente alla realtà vissuta dai residenti della borgata.

La borgata di Tormarancia, nei primi anni Trenta del Novecento, nasceva su quelli che erano stati i terreni della tenuta omonima del Conte De Merode, in un'area non priva di accampamenti e baracche i cui inquilini avrebbero popolato in seguito le case del nuovo quartiere. Molti venivano dalle baracche della borgata laurentina, situata nell'area in cui si costruì il quartiere denominato Montagnola, ma alcune erano presenti anche in via Girolamo Benzoni alla Garbatella sui terreni delle Ferrovie dello Stato, in via di Grotta Perfetta o a Porta San Paolo o sulla via Ostiense dove gli

\footnotetext{
${ }^{1}$ MORAVIA, Alberto, Racconti Romani, Milano, Bompiani, 2008, p. 88.
} 
occupanti delle baracche erano definiti cicoriari e colonia abruzzese. Manufatti di ogni tipo di materiale si potevano scorgere nelle vicinanze, dalla legna al tessuto per tende, fino alla muratura.

Poco distante dalla Garbatella, ma di differente genesi e forma architettonica, Tormarancia può divenire un caso di studio ottimale per approfondire il tessuto sociale in borgate romane ancora poco studiate e approfondite nel loro genesi, nello sviluppo, nella loro evoluzione. Conosciuto ai più ad oggi come il quartiere della street art, in seguito all'intervento artistico "Big City Life" ${ }^{2}$, che ha visto diversi artisti internazionali dare un nuovo volto ad uno dei lotti di edilizia popolare in un'ottica di nuova riqualificazione, Tormarancia non ha vissuto la stessa fortuna di studi ed approfondimenti dei quartieri limitrofi: basti pensare alla già citata Garbatella ${ }^{3}$, al quartiere San Paolo o a San Saba per rendersi conto della differenza di attenzione in merito alle vicende che hanno portato alla costruzione della borgata. Coprendo un arco cronologico che parte dagli ultimi anni Venti ed arriva al finire degli anni Trenta, l'obiettivo dell'articolo è tracciare un primo percorso di indagine e studio in merito alle motivazioni alla base della nascita della borgata, conoscere la provenienza dei primi residenti e descriverne le condizioni abitative e sociali.

Per farlo è doveroso ricostruire sommariamente le vicende che portarono alla costruzione delle prime borgate governatoriali, una delle politiche fasciste dei primi anni Venti che, come ricorda Villani, non fu tra le azioni più propagandate ${ }^{4}$, benché fosse l'avvio di un nuovo sviluppo sociale di grandi porzioni per la città. Di particolare interesse è la costruzione delle cosiddette borgate di «seconda generazione»" ${ }^{5}$ tra le quali Insolera annovera Tormarancia e Primavalle. La costruzione dei ricoveri e delle casette rapide da parte del Governatorato di Roma iniziò nel 1929, gli alloggi erano destinati alle famiglie sfrattate a seguito delle opere di urbanizzazione legate al nuovo piano regolatore e a nuclei familiari che vedevano le proprie abitazioni di fortuna abbattute. Successivamente in questi locali furono alloggiati gli abitanti delle baracche e dei villaggi “abissini”". Il 12 settembre del 1927 il Prefetto del Regno, Segretario Generale del

\footnotetext{
${ }^{2}$ MEZZANO, Francesca, ANTONELLI, Stefano, Big city life: TorMarancia. Gli abitanti di Tor Marancia incontrano 22 artisti venuti da 10 paesi per dipingergli il quartiere, Roma, Lit, 2015.

${ }^{3}$ Per approfondimenti si propone la lettura di: SINATRA, Monica, La Garbatella a Roma 1920-1940, Milano, Franco Angeli, 2006; ROMANA STABILE, Francesca, La Garbatella a Roma: architettura e regionalismo, Roma, Librerie Dedalo, 2012.

${ }^{4}$ VILLANI, Luciano, Le borgate del fascismo. Storia urbana, politica e sociale della periferia romana, Milano, Ledizioni, 2012, p. 51

${ }^{5}$ INSOLERA, Italo, Roma moderna. Da Napoleone I al XXI secolo, Torino, Einaudi, 2011.

${ }^{6}$ Venivano chiamati villaggi abissini gli agglomerati di baracche e case di fortuna per lo più presenti in zone di Roma come Ponte Milvio (abbattuti nel 1925) e Portonaccio (abbattuti nel 1928). La denominazione, affibbiata a questi luoghi dal fascismo, evoca «l'immagine di un nemico da combattere», come se gli abitanti delle baracche dovessero essere schiacciati in modo quasi militare, come se ci fosse una differenza di razza. Cfr. CLEMENTI, Alberto, PEREGO, Francesco (a cura di), La metropoli "spontanea"- Il caso Roma, Bari, Dedalo, 1983.
} 
Governatorato, scriveva ${ }^{7}$ ad Agostino De Pretis, delegato all'assistenza sociale, riguardo al piano di demolizione delle baracche realizzato dall'ufficio preposto: l'abbattimento doveva andare di pari passo con la costruzione di alloggi popolari da assegnare a chi era stato cacciato dalle baracche abbattute. Inoltre le demolizioni dovevano seguire la gradualità dell'attuazione del Piano Regolatore. Alberto Calza Bini, presidente dell'Istituto Case Popolari (ICP) valutava favorevolmente l'inizio delle demolizioni dalla zona centrale della città, in modo specifico da via dei Cerchi, limitrofa al Circo Massimo e al Colosseo. Tutto ciò, in linea con la dualità evidente nella progettazione urbanistica fascista, fondata su criteri opposti nel trattamento del centro della città rispetto allo sviluppo periferico, privilegiando la prima e nascondendo le gravi mancanze della seconda ${ }^{8}$.

Il bisogno di stabilire i criteri con i quali si sarebbero dovuti allontanare i non residenti e come doveva gestirsi la loro riallocazione dopo la demolizione delle baracche e dopo gli sfratti emerse subito per il Governatorato: ci si apprestava ad avviare procedimenti complessi e difficoltosi da gestire per quanto riguardava l'ordine pubblico in città. Il primo intervento a favore degli sfollati fu l'utilizzo dell'ex caserma di viale Angelico, di esigue dimensioni rispetto al numero dei bisognosi. Il Governatorato era pronto a stanziare 20 milioni per l'ICP al fine di facilitare la costruzione di alloggi unicamente destinati ai baraccati. L'Istituto, definito magistralmente da Vidotto «un elemento formidabile di controllo politico e di costruzione del consenso»" svolse un ruolo centrale nelle politiche fasciste a Roma, divenendo il braccio d'azione della mente del Governatorato.

Una memoria di Giunta del 1924 riporta chiaramente le cause dell'emergenza abitativa romana, tra cui la mancanza di alloggi popolari e l'aumento degli affitti:

È diffusa nell'animo delle classi popolari e specialmente di quelle di provenienza rurale l'avversione contro le spese per l'abitazione. Abituate nei paesi d'origine a non pagare affitto di casa poiché anche i più poveri posseggono un umile tugurio, non si adattano a corrispondere una pigione che è ritenuta un balzello insopportabile. [...] Oltre a ciò il miraggio di circondare la baracca di qualche metro di terreno coltivabile a orto, la possibilità di impiantare un gallinaio o altro allevamento di bestiame da cortile, costituiscono per questa gente oriunda dalla campagna un incentivo a rompere ogni contatto con le consuetudini della vita urbana così da spingerla a gruppi verso la periferia per costruirsi il desiderato alloggio ${ }^{10}$.

\footnotetext{
${ }^{7}$ Archivio Storico Capitolino (ASC), Ufficio Assistenza Sociale, Carteggio con titolario, classe 7, baraccati, b. 90, f. 1.

${ }^{8}$ CIUCCI, Giorgio, Gli architetti e il fascismo. Architettura e città 1922-1944, Torino, Einaudi, 1989, p. 80.

${ }^{9}$ VIDOTTO, Vittorio, Roma Contemporanea, Bari, Laterza, 2006, p. 197.

${ }^{10}$ Ibidem.
} 
Ma quali erano le problematiche che si incontravano nel cercare un alloggio? Risulta di grande utilità la lettura della richiesta d'aiuto arrivata all'Assistenza Sociale da un gruppo di famiglie di baraccati oramai da tempo stanziali sulle rive del Tevere presso la via Portuense:

è mai tollerabile e possibile che proprio nell'avvicinarsi della stagione fredda si vogliano mettere in mezzo alla strada e sfrattare tanta famiglie che con tantissimi sacrifici avevano potuto crearsi (senza aiuto di nessuno) con i propri mezzi delle decenti baracche in muratura e in legno ${ }^{11}$ ?

I mittenti della lettera avevano ottenuto i terreni da un proprietario che a sua volta li aveva ricevuti in affitto dal Demanio dello Stato, una situazione non rara da quello che si può leggere nelle carte dell'Assistenza Sociale in cui viene descritto il fenomeno. A Roma molti erano gli speculatori pronti ad approfittarsi della situazione di emergenza ed indigenza dei senzatetto, una piaga in più che si aggiungeva alla già complessa situazione dell'assistenza sociale. Villani ha sottolineato il sovrapporsi di fenomeni quali l'immigrazione, il disagio sociale, gli sfratti, gli sbaraccamenti, con la crisi economica che si abbatté sulla città negli anni Trenta ${ }^{12}$. Le prime avvisaglie di diffuso malcontento, dovuto prioritariamente all'assenza di vere e proprie politiche abitative, si erano palesate già nei primi anni del Novecento. Igiene, indisponibilità di acqua potabile, affollamento erano solo alcuni dei problemi che gli abitanti di Roma si trovavano ad affrontare ${ }^{13}$. Ma qual era il ruolo dell'Ufficio Assistenza Sociale? In un quadro così complesso risultò fondamentale la sua creazione: istituito nel 1926 per rispondere ai bisogni basilari di assistenza della popolazione presente a Roma, l'ufficio conquistò una propria autonomia solo nel 1928, per poi essere soppresso nel 1935 con una suddivisione dei suoi compiti tra i più diversi uffici della macchina capitolina. I compiti di questo ufficio spaziavano dal controllo sull'edilizia assistenziale a quello sulla beneficenza, le opere pie e l'assistenza scolastica e l'infanzia ${ }^{14}$.

Descritti come agglomerati insalubri, le baracche spaventavano per le possibili infezioni e malattie che vi si potevano annidare e dalle quali si potevano diffondere. Nel 1920 l'Ufficio Municipale per il lavoro del Comune di Roma, descrivendo i numerosi agglomerati di baracche presenti in città, prevedeva, ottimisticamente, che a seguito della costruzione di case popolari da parte del Governatorato l'abbattimento delle baracche sarebbe stato immediato ed il problema sarebbe stato risolto ${ }^{15}$. Nonostante i buoni auspici degli uffici comunali, al 1924 la situazione non

\footnotetext{
${ }^{11}$ Ibidem.

${ }^{12}$ VILLANI, Luciano, op. cit., p. 55.

${ }^{13}$ VIDOTTO, Vittorio, op. cit., p. 130.

${ }^{14}$ Per approfondimenti si propone la lettura di GORI, Patrizia (a cura di), L'assistenza sociale negli anni del Governatorato di Roma. L'inventario dell'Ufficio Assistenza Sociale (1926-1935), Roma, Viella, 2016.

${ }^{15}$ UFFICIO MUNICIPALE PER IL LAVORO DEL COMUNE DI ROMA (a cura di), Il problema edilizio, Roma, Tip. Centenari, 1920.
} 
era migliorata, infatti in una lettera del settembre dello stesso anno inviata dall'Assistenza Sociale al Prefetto della Provincia di Roma si evidenziavano ancora gli annosi problemi delle baracche, descritte come "agglomerati che costituiscono pericolosi fomiti di infezioni» ${ }^{16}$. Sulla rivista «Capitolium», nel 1931 venne pubblicato un articolo dal titolo «Delenda baracca!» nel quale si descrivono i baraccamenti come luoghi in cui si annidavano $\mathrm{i}$ «germi del vizio e del delitto» e che assediavano Roma come «cenci pestilenti» ${ }^{17}$. È chiara la considerazione che il Governatorato e la dirigenza fascista avevano per gli abitanti delle baracche, visti come soggetti non in grado di trovare un lavoro, mantenerlo e vivere a Roma secondo la visione di dignità che si imponeva alle fasce più deboli della società. Da una lettura delle carte dell'Assistenza Sociale si possono trarre informazioni utili rispetto allo stato in cui versavano baracche e baraccati a Roma, ricostruzioni non certo scevre da giudizi sommari rispetto alle condizioni di vita e l'attitudine al lavoro:

Il problema è stato ed è oggetto di continuo, attento studio. Occorre aiutare, per il minimo indispensabile, questi disgraziati, per la maggior parte non grati alla R. Questura; ma sarebbe estremamente pericoloso, ai fini sociali, addolcire, anche di poco, le attuali loro condizioni di vita. Perché, dato che queste masse, salvo poche e precarie situazioni, sono costituite da disoccupati per cialtroneria o incapacità, qualunque piccolo allentamento, fornito ad individui proclivi all'ozio, non servirebbe ad altro che ad accentuarne la neghittosità e ad attivare il fenomeno immigratorio dei non desiderabili e contro il quale si esperimenta la diuturna attività della Pubblica Sicurezza ${ }^{18}$.

Ma come avveniva la demolizione di questi insediamenti? Nel giugno del 1933 la Ripartizione V con una lettera ${ }^{19}$ «urgentissima» scriveva alla direzione dell'Assistenza Sociale per comunicare che la procedura di abbattimento delle baracche sino ad allora utilizzata doveva essere cambiata, visto l'ingente numero di baracche da abbattere e la lentezza della procedura stessa. Si chiarisce che visto l'interesse di «risanamento morale oltre che igienico di interi quartieri della città» ${ }^{20}$, la Questura doveva occuparsene prioritariamente. L'avvocato a cui il Governatorato aveva chiesto una consulenza sul tema consigliò di farsi rilasciare da parte degli sbaraccati una dichiarazione contenente la rinuncia a eventuali indennizzi e l'accettazione di un alloggio o di un sussidio. Questa decisione però non venne condivisa dall'Assistenza Sociale poiché «molti degli sbaraccati potranno rifiutare tale dichiarazione, che non potrà essere imposta per mancanza di sanzioni, sta

\footnotetext{
${ }^{16}$ ASC, Ufficio Assistenza Sociale, Carteggio con titolario, classe 7, baraccati, b. 91, f. 1 .

${ }^{17}$ ZUCCA, G., «Delenda baracca!», in Capitolium, VII, 1/1931, pp. 44-48, p. 44.

${ }^{18}$ ASC, Ufficio Assistenza Sociale, Carteggio con titolario, classe 6, Ricoveri, b. 84, f. 3.

${ }^{19}$ Ibidem.

${ }^{20}$ Ibidem.
} 
di fatto che la predetta richiesta non farà che mettere in sospetto gli interessati e provocare da loro parte le resistenze che si vogliono evitare» ${ }^{21}$.

La stima complessiva fatta vedeva il numero delle famiglie da rimpatriare oltre le 1.500 unità, per le quali si prevedeva una spesa di 600 lire a famiglia. In tutta Roma negli alloggi dedicati agli sbaraccati venivano accomodate 2.173 famiglie pari a 11.619 persone. Nel 1933 le baracche abbattute furono 1.080 , quelle ancora da abbattere 2.920. Tra le famiglie sbaraccate 408 potevano essere rimpatriate ma fino a quel momento di rimpatri ne erano stati effettuati solo 170 per un totale di 600 persone. Dall'elenco ${ }^{22}$ delle provenienze dei baraccati di Ostia rimpatriati vediamo che 10 di loro venivano da Bari, 6 da Nuoro, 4 da Sassari, e c'è anche chi veniva da Buenos Aires. Dalle schede personali si può vedere che la loro precedente abitazione era sempre una baracca situata o in via della Magliana o in Corso Regina Margherita sempre a Ostia, demolita poco prima. C'era anche chi precedentemente abitava tra le dune di Ostia.

Al 25 novembre 1931 il numero di baracche da demolire era pari a 1.846 unità nella zona interna della città (il limite corrisponde alla vecchia cinta daziaria), 1.989 nel suburbio, ovvero la zona media, 907 nell'agro romano, ovvero la zona esterna. Le previsioni del 1920 rispetto alla soluzione del problema sembrano ormai un lontano ricordo. Questi sono gli anni delle nuove costruzioni a Monte Sacro, Garbatella, Maranella e Ponte Milvio realizzate dall'ICP, ma anche quelli che segnano la nascita degli Alberghi Suburbani (sempre a Garbatella) in cui potevano alloggiare fino a 900 famiglie $^{23}$ e che rappresentarono una soluzione temporanea strategica per la gestione dell'emergenza, pur avendo anch'essi più di una pecca, a partire dall'affitto proibitivo di locali pari a 4,50 lire al giorno, non comprensivi di pasti da consumarsi a pagamento o nei refettori o in trattorie convenzionate ${ }^{24}$.

Gli anni che vanno dal 1930 al 1933 sono stati anni complessi nella gestione dell'emergenza abitativa. Sia per i fenomeni migratori, sia per la scossa urbanistica data dal fascismo alla Roma postunitaria, nacquero borgate quali Acilia, Donna Olimpia, Tufello, Val Melaina e, ovviamente, Tormarancia. Inoltre vedono la luce la semirurale borgata San Basilio, la borgata Gordiani, Prenestina, Pietralata. Risulta più chiaro il quadro dell'attività edilizia messa in essere se si leggono i dati tra il 1924 ed il 1941: la produzione media stimata è pari a 10 mila alloggi l'anno ${ }^{25}$. Si stima che il solo abbattimento delle case dei quartieri Borghi e Spina abbia portato alla

\footnotetext{
${ }^{21}$ Ibidem.

${ }^{22}$ Ibidem.

${ }^{23}$ BERLINGUER, Giovanni, DELLA SETA, Roberto, Borgate di Roma, Roma, Editori Riuniti, 1960, p. 94.

${ }^{24}$ LUNADEI, Simona, La cura dell'infanzia negli anni del Governatorato, in GORI, Patrizia (a cura di), L'assistenza sociale negli anni del Governatorato di Roma. L'inventario dell'Ufficio Assistenza Sociale (1926-1935), Roma, Viella, 2016, pp. 9-134, p. 25.

${ }^{25}$ MAROI, Lanfranco, «L'attività edilizia in Roma nel quinquennio 1934-1938», in Capitolium, XIV, 6/1939, pp. 268-290, p. 271.
} 
distruzione di 729 appartamenti e alla cacciata di 4.992 persone in cerca di ricollocazione ${ }^{26}$. Il censimento del 1931 documenta chiaramente la crescita urbana figlia delle politiche fasciste: rispetto al 1921 si segnala una crescita del 373\% delle case nell'agro e nel suburbio ${ }^{27}$, il dato di popolazione presente a Roma era pari a $1.008 .083 \mathrm{abitanti}^{28}$. La distribuzione della popolazione nel Lazio al 1931 descrive chiaramente quanto Roma fosse un attrattore senza paragoni: Roma e la sua provincia accoglievano il $61 \%$ della popolazione regionale, quasi il $9 \%$ nella provincia di Viterbo, il $16 \%$ in quella di Frosinone, quasi il 7\% in quella di Rieti e in quella di Latina ${ }^{29}$.

Nonostante le difficoltà in cui la città si dibatteva tra la fine degli anni Venti e l'inizio degli anni Trenta, il 28 ottobre del 1930 fu presentato il nuovo piano regolatore: una città bisognosa di programmazione e sviluppo si affidò ad interpreti del tempo come Marcello Piacentini per pensarsi nel futuro ${ }^{30}$. Esplicativo dello spirito urbanista del tempo è il discorso pronunciato da Mussolini al Senato il 18 marzo 1932, in cui compare la metafora di «S.M. il piccone» ${ }^{31}$ e che così spiegava le migliorie per la città previste dal nuovo piano regolatore:

Spostando la popolazione verso i colli o verso il mare, noi effettuiamo il disistipamento di Roma, demoliamo tutte le casupole infette, facciamo i diradamenti necessari a tutti i fini, diamo del sole, della luce, dell'aria al popolo ${ }^{32}$.

Non erano previsti dal Governatorato solo gli sbaraccamenti, ma si ipotizzava anche la chiusura dei ricoveri, oramai «eccessivamente densi» ${ }^{33}$ e possibili ricettacoli insalubri. Chi trovava riparo nei ricoveri spesso veniva allontanato per diverse motivazioni: dall'elenco dei trasferimenti dal ricovero Casermette si viene a conoscenza di alcuni baraccati allontanati per immoralità come successe ad Antonietta Careddu, o per indisciplina recidiva come per Iori Cataldo, o per morosità come per Pietro Mancini.

Come potevano conquistare un vano per vivere coloro che venivano sbaraccati e non venivano rimpatriati? Tutti potevano far richiesta per le casette allestite dal Governatorato, ma, una volta ottenuto l'alloggio, bisognava rispettare chiare regole stabilite dal Servizio Alloggi del Comune tra cui emblematica è la raccomandazione di «denunciare subito all'Ispettore i casi di malattia

\footnotetext{
${ }^{26}$ CEDERNA, Antonio, Mussolini urbanista. Lo sventramento di Roma negli anni del consenso, Roma-Bari, Laterza, 1979 , p. 222.

${ }^{27}$ CLEMENTI, Alberto, PEREGO, Francesco (a cura di), op. cit., p. 387.

${ }^{28}$ BERLINGUER, Giovanni, DELLA SETA, Roberto, op. cit., p. 171.

${ }^{29}$ Ibidem, p. 23.

${ }^{30}$ PIACENTINI, Marcello, Relazione programma a S.E. il capo del governo sul progetto del piano regolatore di Roma, Roma, Canella, 1930.

${ }^{31}$ MUSSOLINI, Benito, La Roma di Mussolini (18 marzo 1932), in ID., Le opere i discorsi e gli scritti (1914-1942), URL:

< http://www.adamoli.org/benito-mussolini/pag0493-04.htm > [consultato il 29 giugno 2017].

${ }^{32}$ Ibidem.

${ }^{33}$ Ibidem.
} 
contagiosa ed attenersi alle norme igieniche che in tali circostanze venissero impartite» ${ }^{34}$. Risulta interessante questa azione di prevenzione perché dà un'idea di quella che era la situazione igienico-sanitaria dei baraccati, ritenute figure al limite e malsane che andavano tenute sotto controllo.

\section{Dalle baracche e i ricoveri a Tormarancia: la nascita di una borgata}

Tormarancia non era ubicata in un luogo privo di accampamenti e baracche. Infatti, anche nelle aree limitrofe alle nuove casette rapide del Governatorato sorgevano baracche i cui inquilini avrebbero popolato in seguito le case della nuova borgata. Inoltre, i terreni su cui fu costruita Tormarancia erano vicinissimi alle "case rapide" di via delle Sette Chiese, sorte nel 1930, limitrofe all'omonimo ricovero e destinatarie di molti degli abitanti delle baracche abbattute nel 1931. Risultano didascaliche le lamentele sullo stato di queste casette rapide avanzate al Governatorato da alcuni abitanti solo ad un anno dalla costruzione della borgata:

Sono veri porcili, indegni di una nazione civile e di Roma. Gabinetti in comune, il fetore fa retrocedere, lavandini idem ove tutti si lavano la persona e le stoviglie, cucinare in camera ove si dorme intere famiglie di sei e più persone, i figli sono abbandonati alla strada per giornate intere perché la lontananza non permette alle mamme di condurli in città $e$ nell'impossibilità di farli uscire dal fango e da rompicolli [...]. Così tutti questi figli saranno i futuri abitatori delle patrie galere ${ }^{35}$.

Per molto tempo le casette di Sette Chiese versarono in condizioni deplorevoli: senza luce, con ingenti danni alle strutture, cadute di calcinacci e acqua piovana che filtrava nelle stanze. Tutte le mancanze venivano segnalate dai custodi, inascoltati. Impossibile mettere fioriere ad abbellire le terrazze: nel 1931 vennero vietate perché il peso le rendeva pericolose per la struttura. C'erano anche inquilini che, mal sopportando lo stato di degrado in cui si trovava il ricovero Sette Chiese, si appellavano in modo accorato al Governatore richiedendo diritto alla dignità e all'igiene ${ }^{36}$. Gli abitanti del ricovero Sette Chiese arrivarono a richiedere al Governatorato di svolgere il lavoro di scopini, come descritto dalle richieste di assunzione presenti in alcuni fascicoli ${ }^{37}$ dell'Assistenza Sociale che raccontano di candidati in situazioni familiari e sociali al limite, con membri della famiglia affetti da gravi malattie o loro stessi infortunati dai lavori precedentemente svolti.

\footnotetext{
${ }^{34}$ Ibidem.

${ }^{35}$ ASC, Ufficio Assistenza Sociale, Carteggio con titolario, classe 6, Ricoveri, b. 80, f. 8.

${ }^{36}$ Ibidem

${ }^{37}$ Ibidem.
} 
Vari sono i passaggi che portarono, nel 1933, alla costruzione della borgata di Tormarancia: le diverse vicende delle politiche abitative romane si intrecciano inevitabilmente con la sua storia. Dagli sventramenti del centro città agli sfratti dovuti allo sblocco delle locazioni e degli affitti del 1930, si aprì la strada alla costruzione di borgate come Pietralata, Gordiani, Acqua Brulicante e Tormarancia. Al 19331.500 famiglie erano state ricollocate in nuove strutture, ma ne vennero censite altre 962 collocate ancora in baracche o giacigli di fortuna ${ }^{38}$.

Stando ai dati esposti, potremmo chiederci come nasce una borgata e di quali servizi bisognava dotarla. I lavori per la costruzione della nuova borgata andarono dal maggio ai primi giorni dell'agosto 1933. Durante questi mesi il Governatorato concesse alla Società Autonoma Fratelli Giovannetti l'incarico di costruire case e negozi a Tormarancia, collocando questi ultimi nei locali per sfrattati «per corrispondere alle normali esigenze delle famiglie ivi ricoverate»" ${ }^{39}$ É una concreta testimonianza della attività commerciali presenti nella borgata anche la richiesta di Alfredo Matteini, invalido di guerra e inquilino delle nuove case di Tormarancia, per l'apertura di un piccolo vano da barbiere così da poter pagare il fitto dell'alloggio. Se è vero che nel 1933 si dava l'avvio alla costruzione di negozi, dal carteggio 40 tra l'ufficio della Ripartizione VII e l'Assistenza Sociale si viene a conoscenza di un'ampia rete di vendita abusiva di ortaggi, frutta, verdura radicata tra gli abitanti della borgata.

Nel 1935, anno in cui la borgata fu presa in gestione dall'ICP, la stessa Società fu incaricata della sistemazione dei canali di scolo e dei lavori di ampliamento di fognature, cucine e recinzioni da effettuarsi presso il Giardino d'Infanzia di Tormarancia, a causa dell'aumento del numero di bambini che lo frequentavano. Altre informazioni sui luoghi in cui tale borgata stava nascendo li restituisce una nota della Ripartizione V che riporta le lamentele della ditta Giovannetti riguardo i danni causati da alcuni buoi al pascolo nell'area occupata dalle casette rapide ${ }^{40}$. La presenza di un pascolo tra le aree in via di edificazione è emblematica di quello che, fino a poco prima, erano questi luoghi, ovvero campagne in cui la pastorizia era una delle principali attività insieme alla coltivazione a vigna. Il bestiame risultava di proprietà dell'ex affittuario del fondo sul quale si stava costruendo, che, anche se allontanato dal personale della ditta, non demordeva nel voler tenere i buoi nei luoghi da lui sempre utilizzati.

Di fondamentale importanza per questa nuova borgata erano i luoghi di aggregazione per i bambini. È del 3 marzo 1934 la deliberazione del Governatorato di Roma per la concessione della gestione delle Case dei bambini in località Gordiani e Tormarancia al Comitato Autonomo Asili Agro Romano. L'obiettivo del Governatorato era quello di estendere ed intensificare i servizi assistenziali nelle borgate popolarissime come Gordiani e Tormarancia. Per questo vennero

\footnotetext{
${ }^{38}$ LUNADEI, Simona, op. cit., p. 27.

${ }^{39}$ ASC, Ripartizioni, Ripartizione V, Lavori Pubblici, Ragioneria appalti esauriti, b. 141, f. 251.

${ }^{40}$ Ibidem.
} 
costruiti ampi edifici per accogliere i bambini con lo scopo di «sottrarli ai pericoli della strada e della convivenza $»^{41}$. L'istruzione impartita nelle Case dei bambini era quella preelementare ed era previsto anche un servizio mensa dove, si legge, «sarà somministrata la refezione calda meridiana e una seconda merenda asciutta» ${ }^{42}$. Inoltre nelle strutture venivano distribuiti ai più bisognosi indumenti e scarpe, grembiuli e cappellini. La conclusione dei lavori di costruzione degli edifici era prospettata per metà marzo dello stesso anno. Da una lettera del luglio 1934 del Governatorato all'Impresa Giovannetti si comprende lo stato delle aree antistanti alle scuole, infatti viene richiesto alla ditta un lavoro aggiuntivo che comprenda anche la realizzazione di marciapiedi fuori dagli edifici, non previsti nel progetto iniziale, come anche una recinzione e delle docce, di cui i bagni erano sprovvisti ${ }^{43}$.

Da uno scritto dell'Ufficio Assistenza Sociale indirizzato a Edmondo Rossoni, ministro dell'Agricoltura e Foreste e Sottosegretario alla Presidenza del Consiglio, si può leggere un resoconto dettagliato riguardo i luoghi di aggregazione e i servizi dedicati al quartiere, tra cui era presente anche un chiesa dotata di «ambienti adatti alla esplicazione di attività assistenziali» ${ }^{44}$. Inoltre la borgata era stata dotata di una sede del Fascio destinata alla Federazione dell'Urbe, e di una grande piscina per i bambini per fargli praticare «l'idroterapia, il nuoto, la ginnastica, e la elioterapia» ${ }^{45}$.

L'idea della costruzione di una chiesa nasceva dalle richieste degli abitanti, una per tutti Ippolita Segatori Antico che, insieme ad un gruppo di donne, scriveva alla Segreteria del Governatorato chiedendo la costruzione di un luogo di culto:

Voglia accordarci una chiesina, non chiediamo una chiesina con una bella estetica, ma solamente una misera Baracchetta onde poter assistere a qualche funzione religiosa nella quale potranno andare anche vecchi e bambini senza camminare tanto e anche inciviliggire [sic] tante persone ${ }^{46}$.

Da una nota ${ }^{47}$ dell'Assistenza Sociale alla II Ripartizione Patrimonio del 28 giugno 1934 si apprende che la richiesta degli abitanti era stata accolta e che si era tenuto già un primo sopralluogo nell'area da destinarsi, ma, in questi luoghi, venivano trovate costruzioni volte a pubblici esercizi, proprio a fianco alla Casa dei Bambini. Dopo aver chiesto l'abbattimento dei manufatti, si provvedeva a cominciare i lavori per la costruzione della chiesa.

\footnotetext{
${ }^{41}$ ASC, Ufficio Assistenza Sociale, carteggio con titolario, classe 3, Istituzioni di assistenza, b. 32, f. 2.

${ }^{42}$ Ibidem.

${ }^{43}$ ASC, Ripartizioni, Ripartizione V Lavori Pubblici, Ragioneria Appalti Esauriti, b. 196, f. 367.

${ }^{44}$ ASC, Ufficio Assistenza Sociale, Carteggio con titolario, classe 6, Ricoveri, b. 84, f. 3.

${ }^{45}$ Ibidem.

${ }^{46}$ Ibidem.

${ }^{47}$ Ibidem.
} 
Riguardo alle sedi degli organi fascisti il Segretariato Generale sottoponeva nell'aprile del 1934 all'Assistenza Sociale una richiesta di concessione di locali a Tormarancia per l'Opera Balilla locale, indicando come idonei due vani in un villino di via delle Sette Chiese. Alla richiesta è allegata la nota del Presidente Provinciale dell'Opera Balilla, Orfeo Santi, che sottolineava l'importanza della concessione di questi vani almeno fino all'apertura di una «Casa Balilla che renda possibile la soluzione del problema educativo, ginnico e culturale della interessante e bisognosa zona di Tormarancio» ${ }^{48}$.

Dal gennaio al marzo 1935 furono continue le problematiche sottoposte al Governatorato, dalla manutenzione dei tetti delle case alla sistemazione della stessa Casa dei bambini che, seppur di nuova costruzione, presenta subito necessità di manutenzione; venne richiesta la sistemazione dei servizi igienici delle case dei custodi, ma anche l'attenzione alla salubrità delle strade, compromessa dalle pozzanghere create da fontanelle mal funzionanti. Nelle prime settimane del 1935 è l'Ufficio fogne a lamentare la poca pulizia nelle cunette che fungono da collettori: l'immondizia ivi depositata impediva il normale flusso delle acque e lo stato dei luoghi portò l'Ufficio a descrivere il servizio di pulizia come «completamente abbandonato» ${ }^{49}$.

Nell'aprile del 1935 venne redatto il verbale di consegna di abitazioni nella «borgata popolare per ricovero sfrattati in località Sette Chiese» ${ }^{50}$ per 732 famiglie con nuclei familiari sporadicamente inferiori alle 7 unità, arrivando a contare in alcuni casi sino a 12 persone. Molte famiglie arrivavano nella nuova borgata, che però aveva un unico ambulatorio sanitario, sprovvisto di telefono. Non un problema da poco per l'Ufficio igiene del Governatorato che richiese l'istallazione dell'impianto di posta telefonica visti i «continui e urgenti servizi sanitari $»^{51}$. Da una nota ${ }^{52}$ dello stesso ambulatorio al Segretario Generale nel 1933 si apprendono le precarie condizioni di lavoro del personale, che, in attesa di locali da occupare per la notte, dovevano dormire lontani tre chilometri dalla borgata. Una successiva nota del settembre 1933 segnala le dimissioni rassegnate dagli stessi lavoratori dell'ambulatorio di Tormarancia e di via Teano, per le condizioni di lavoro particolarmente difficili a cui dovevano sottostare, quali anche la custodia degli ambienti e dei ferri da lavoro, mansione che rendeva impossibile allontanarsi dalla borgata e tornare alla propria casa tra un turno e l'altro ${ }^{53}$. Era presente nella struttura anche una profilassatrice già operante nell'Agro Romano, di servizio sia a Tormarancia sia in via Teano. Un infermiere in pianta stabile a Tormarancia però c'era, Ubaldo Coretti, costretto richiedere il trasferimento dalla sua abitazione nella borgata vista l'umidità che nuoceva gravemente allo stato

\footnotetext{
${ }^{48}$ Ibidem.

${ }^{49}$ ASC, Ufficio Assistenza Sociale, carteggio con titolario, classe 6, Ricoveri, b. 88, f.3.

${ }^{50}$ Ibidem.

${ }^{51}$ Ibidem.

${ }^{52}$ ASC, Ripartizioni, Ripartizione VIII, Igiene e Sanità (1871-1940), Carteggio, Serie II, b. 73, f. 9.

${ }^{53}$ Ibidem.
} 
di salute del figlio. Alle difficili condizioni di lavoro del personale sanitario si associava una situazione igienica precaria, che portò anche al ripresentarsi di episodi malarici: da giugno a settembre 1934 tre casi si registrarono a Tormarancia e quattro negli Alberghi della Garbatella ${ }^{54}$.

La borgata era provvista di sedi scolastiche, ma, nell'agosto del 1935, l'Ufficio Assistenza Sociale informò il Provveditore per le scuole del Governatorato che lo stato dei luoghi era compromesso:

Com'è noto alla s. v. illustre, in seguito a richiesta di codesto ufficio, nei primi del corrente anno, vennero adattati per usi scolastici alcuni locali nella borgata Tormarancia. Tali locali, peraltro, non sono mai stati utilizzati e incustoditi sono continuamente soggetti alle devastazioni delle ragazzaglie del luogo ${ }^{55}$.

L'immagine che questa documentazione ci restituisce degli abitanti del luogo è ben connotata, sia dal termine ragazzaglie sia dai motivi e dagli obiettivi di presa in custodia dei bambini da parte del Comitato Autonomo Asili Agro Romano, come il toglierli dalla strada o dalla convivenza.

Ma chi erano in realtà gli abitanti della nuova borgata? Al 14 febbraio del 1934 nella borgata popolarissima di Tormarancia erano presenti 502 famiglie pari a 2.631 persone temporaneamente ospitate a canone gratuito, di cui 194 famiglie provenienti da Ricoveri, 272 dalle baracche, 31 da sfrattati privati, 5 sfrattati dall'Istituto Case Popolari. Le abitazioni di provenienza dei nuovi inquilini delle case di Tormarancia erano principalmente il Pastificio Costa, le baracche di via Acqua Acetosa, quelle di via Principe Amedeo, quelle limitrofe ai villini di via delle Sette Chiese, le baracche nella Garbatella, o nei pressi di Stazione San Pietro e di Primavalle.

Gli abitanti della borgata erano, per la maggior parte, nati a Roma, ma troviamo censiti anche inquilini provenienti da Norcia, dal viterbese, dal reatino, Terni, Guspini nel cagliaritano, Mantova, Napoli, Perugia: tutto il centro Italia è ben rappresentato.

Una guida fondamentale per comprendere la realtà sociale che si stava formando nella nuova Borgata Popolarissima è fornita dalle schede ${ }^{56}$ personali degli inquilini, redatte a partire dal luglio del 1933 dall'Ufficio Assistenza Sociale, dalle quali si possono trarre svariati e importanti dati riguardo la vita di queste persone. In primo luogo l'attività lavorativa svolta dagli inquilini: gli uomini erano principalmente facchini, muratori, manovali, meccanici e, per quanto riguarda le donne, molte erano le sarte. Di certo non tutti i residenti nella borgata lavoravano: al 1935 ben 350 capifamiglia risultavano disoccupati ${ }^{57}$. Nella scheda c'era la possibilità di segnalare anche eventuali problemi con la giustizia, un campo che in molti casi è denso di informazioni. C’è chi

\footnotetext{
${ }^{54}$ BORTOLOTTI, Lando, Roma fuori le mura. l'Agro romano da palude a metropoli, Roma, Laterza, 1988, p. 278.

${ }^{55}$ ASC, Ufficio Assistenza Sociale, carteggio con titolario, classe 6, Ricoveri, b. 88, f. 3.

${ }^{56}$ ACS, Ufficio Assistenza Sociale, Carteggio senza titolario, richieste di ricovero e alloggio, b. 23, f. 1.

${ }^{57}$ FARINA, Milena, VILLANI, Luciano, Borgate romane. Storia e forma urbana, Forlì, Libria, 2017, p. 175.
} 
aveva precedenti penali per immoralità, oltraggio, truffa, liti familiari, violenza, accattonaggio. Le schede inoltre classificavano anche le persone in base all'adesione al fascio e all'essere stati o meno combattenti, le due caratteristiche erano annotate con matita rossa al margine dei campi prestabiliti dalla scheda, un'aggiunta significativa che connotava non poco chi si apprestava a popolare la borgata. Questo è il caso di Lotario Valentini: nel suo fascicolo si trova una scheda del servizio alloggi del 1929 dove viene descritta la sua attività lavorativa e la sua condotta morale. Il Commissariato di San Paolo lo segnalava come "comunista" e da una nota del Sorvegliante del ricovero dove abitava con la famiglia sappiamo che, se ripresi, rispondevano con parole provocatorie e ingiuriose minacce, svelando un temperamento complesso ed una radicata maleducazione, giudizi sicuramente legati alla conquistata nomea di comunista. Come il Valentini, anche Leonida del Giovanni venne attenzionato per "motivi politici”. Altro sovversivo era Egidio Mennini, così descritto dal Gruppo rionale Torpignattara "Raffaele Lulli", sottosezione borgata gordiani, della Federazione Fascista dell'Urbe:

Elementi di sobillazione sociale, recalcitranti a tutti gli ordini e le istituzioni di carattere disciplinare e benefico hanno dimostrato ripetutamente, nonostante i fraterni e continui richiami, poca famigliarità con i vicini e l'assenza completa di doti educative verso dirigenti e comandati al buon andamento, suscitando, con maldicenze, contrasti e disgustosi commenti, fino al raggiungimento della violenza, tali da produrre lesioni ${ }^{58}$.

Dalle carte apprendiamo notizie anche sulla moglie di Mennini, Fernanda Cerroni: «si permetteva in pubblico di fare degli apprezzamenti non tanto benevoli all'indirizzo del fascio. Richiamata al dovere dal personale di servizio, questa rispondeva arrogantemente e non bastando ciò inveiva contro un milite, graffiandolo al viso e scagliava pure un bidone contro il milite» ${ }^{59}$. Il mese successivo a questi avvenimenti, venivano trasferiti entrambi a Tormarancia. Nella lista dei casi complessi da gestire per sorveglianti e forze dell'ordine ci sono comunisti, sovversivi, ma anche casi come quello di Italia Moretti, madre di cinque figli, segnalata nel 1932 dal Custode del ricovero di Via Appia Nuova perché:

Tiene un contegno alquanto indisciplinato e per di più trascura la propria famiglia facendola soffrire per fino la fame. La Moretti ripartisce la spesa di viveri che gli viene data giornalmente e la porta al suo amante che vi abita nel ricovero di via Tuscolana perché trovasi disoccupato, mentre i suoi figli ci sono dei giorni che chiedono il pane ora ad una famiglia ora da un'altra che vi abitano vicino ${ }^{60}$.

\footnotetext{
${ }^{58}$ Ibidem.

${ }^{59}$ Ibidem

${ }^{60}$ Ibidem.
} 
Se il quadro descritto sia oggettivo o meno non è facile a dirsi. Nello scritto veniva enfatizzato anche un suo costante rientro al ricovero fuori orario, intorno alle 22. La figura della donna vedova che nuovamente si accompagna con un uomo pur avendo cinque figli ed una madre a cui badare potrebbe aver alterato i giudizi dei custodi, sottoponendo la Moretti ad un'ingiusta fama.

Andando ad indagare tra le vicende personali degli abitanti del luogo si possono ricavare dati importanti sulla realtà sociale delle borgate del tempo. Umberto Giovagnoli, sfrattato per morosità dagli Alberghi suburbani della Garbatella, aveva costruito nel 1933 una baracca di lamiera e legname a ridosso della Casa dei Bambini limitrofa agli Alberghi, come da nota ${ }^{61}$ dell'ICP. Oppure Gianmarco Guglielmo, milite della $112^{\mathrm{a}}$ Legione, precedentemente residente in via Prenestina, che, oramai senza casa, viene segnalato dal Ministero dell'Interno al Governatorato di Roma e poi in seguito locato in Tormarancia. Paradigmatica dello stato sociale ed economico dei nuovi residenti è la dichiarazione di Eugenio Salvati che chiese:

La bontà di accordarci un vano nella zona di Tormarancia, perché qui non è che non voglio pagare, la pigione è troppo e non posso arrivarci. Qui all'Istituto non mi sente nessuno perché dicono che vani soli non ce ne sono sicché noi dovessimo andare a dormire per strada. Questo è il ricompenzo [sic] che ho fatto quattro anni di Guerra sono decorato con la medaglia d'onore, sono rimasto sordo e con i piedi congelati che in seguito [...] nemmeno riconosciuto avendo dovuto molto girare, allora ero giovane e non pensavo ai disastri a venire perciò mi trovo nella più squallida miseria ${ }^{62}$.

Nei fascicoli personali si possono leggere anche numerose raccomandazioni da parte dell'apparato associativo fascista e dalle sezioni politiche del partito al fine di trovare un alloggio per persone vicine al pensiero fascista, prassi comune al tempo. Ad esempio Tommaso Berettini, proveniente da Primavalle, venne segnalato allo ICP dall'Opera Balilla per ottenere la casa Tormarancia. Lo stesso Berettini descriveva in una lettera la propria condizione:

Il sottoscritto vive in un cantuccio di una stanza nella quale si accende il fuoco, si mangia e ci si sta tutto il giorno in sette perché nell'altra non ci entra che per dormire essendoci tre letti e ci dormono la nipote del Berettini con il marito un figlio di undici anni uno diciotto e la suocera di settantaquattro anni ${ }^{63}$.

\footnotetext{
${ }^{61}$ Ibidem.

${ }^{62}$ Ibidem.

${ }^{63}$ Ibidem.
} 
Per la famiglia del Federato ex Sergente degli Arditi Enrico Bonifazzi era invece la Federazione Nazionale Arditi d'Italia, sezione dell'Urbe, a premurarsi presso l'Assistenza Sociale perché il membro della Federazione trovasse ospitalità a Tormarancia. Stessa sollecitazione venne dall'«Ardito d'Italia, Giornale degli Arditi di tutte le fiamme». Tra i raccomandati c'era anche Vittorio Ambrosini, segnalato dalla Federazione Fascista dell'Urbe, per richiesta del Segretario particolare della Federazione, Ettore Lucarelli. La stessa Federazione, per nome di Vezio Orazi, il Segretario, raccomandò allo ICP anche la vedova Caterina Amore Giovagnoli come persona in "tristissime condizioni economiche» ${ }^{64} \mathrm{da}$ mandare, se possibile, a Tormarancia. Anche l'Associazione Nazionale Combattenti, sezione di Roma, sottosezione Portuense, evidenziava le condizioni di un suo protetto, Bruto Barchi, combattente iscritto a quella sottosezione: abitava in un «misero e antigenico alloggio» ${ }^{65}$ dal quale era stato sfrattato ed era in cerca di un vano nella borgata di Tormarancia.

Per il caso di Angelo Moscato fu il Gruppo "Tito Menichetti" a muoversi per una casa nella borgata: un figlio con ernia inguinale e una moglie, destinati a dormire all'aperto. La lettera di raccomandazione si chiude quasi con una minaccia: «in caso contrario questo Gruppo sarà costretto a segnalare il caso alla Federazione Fascista dell'Urbe. Saluti Fascisti» ${ }^{66}$. La famiglia era stata sfrattata dal ricovero di Sette Chiese poiché solita ad atteggiamenti molesti e che ledevano la quiete pubblica, tanto da portare alla decisione dell'allontanamento ${ }^{67}$.

Anche la Sottosezione Portuense dell'Associazione Nazionale Combattenti aveva i suoi casi da segnalare, tra cui quello del combattente della Prima guerra mondiale Gaetano Patrini e della sua famiglia, in cerca di ricovero, possibilmente a Tormarancia. La situazione malsana dalla quale provenivano toccava tutto il nucleo famigliare, tanto da portare alla morte dell'unico figlio del Patarini, colpito da polmonite ${ }^{68}$.

Dalle raccomandazioni delle varie articolazioni del fascio passiamo alle storie di chi, dichiaratamente fascista, in condizione di disagio, vedeva nel Duce e nelle sue politiche una possibile via d'uscita al degrado in cui viveva. «Fascista leale e sincero», così si definiva Enrico Baldoni in una preghiera per la soluzione della sua situazione abitativa, non consona a suo avviso alla decenza. Nelle sue parole è presente il paradosso che nasce dal confronto tra l'evocazione costante della bellezza e della forza della Roma antica fatta dal fascismo e lo stato in cui molti erano costretti a vivere, ai margini, umiliati:

\footnotetext{
${ }^{64}$ Ibidem.

${ }^{65}$ Ibidem.

${ }^{66}$ Ibidem.

${ }^{67}$ ASC, Ufficio Assistenza Sociale, Carteggio senza titolario, richieste di ricovero e alloggio, b. 24, f. 1.

${ }^{68} \mathrm{ASC}$, Ufficio Assistenza Sociale, Carteggio senza titolario, richieste di ricovero e alloggio, b. 23 , f. 1.
} 
Il grande maestro e filosofo Diogene insegna che l'umigliazione [sic] è dei saggi, ma mi sono già troppo piegato alla umigliazione. Io qui sottoscritto faccio presente che nel Regno Fascista, creatore e arteficie di tante belle cose; mi trovo né la brutimento più mostruoso che mente umana possa immaginare, tutti anno casa, mendicanti, gente della pegiore specie ed io poeta dialettale romano di tante generazione mi tengono nelle più fitte tenebre dell'abrutimento dei tempi. Supestite di una casa che pericolava così mi è data ospitalità nella borgata di Tormarancio ${ }^{69}$.

A Baldoni vennero assegnati due vani nella borgata, in uno dei quali dormivano sei persone e per questo si rivolse al Governatore con parole dure, fondamentali per comprendere lo stato in cui si viveva a Tormarancia e cosa fosse la vita nelle nuove borgate:

dove è l'igiene, la moralità, dove è il pudore. Ed i vecchi che sono arispettati nella Cina e nei paesi più barberi del continente, io vecchio di settantadue anni di età sono già dieci mesi che sto dormendo pertera e chissà quanto ancora dovrò dormire ancora, e questo stato di cose accade nella Roma imperiale faro luminoso e culla della civirtà latina e maestra della gente $[\mathrm{sic}]^{70}$.

\section{Vivere in borgata. Le condizioni di vita nella nuova Tormarancia}

Nella nuova borgata si incrociano vite e storie differenti, accumunate da miseria e difficoltà. Da soggetti «temibili sotto ogni rapporto» ${ }^{71}$, alle donne in grave stato di indigenza, con storie di vita complessa alle spalle come quella di Ida Coronelli, segnalata dal commissariato di Polizia di San Lorenzo per il suo stato di indigenza. Madre di quattro figli, moglie di un carrettiere disoccupato, «versa nella più squallida miseria e per di più senza casa ed è costretta la sera a rifugiarsi con $\mathrm{i}$ figli in qualche portone» ${ }^{72}$. La donna, da quanto riporta la Polizia, era affetta da grave bronchite ma, troppo occupata a pensare al bene dei figli, trascurava se stessa. Dopo l'intervento delle forze dell'ordine venne ospitata a Tormarancia. Una vita di miseria è quella vissuta da Agata De Angelis che, dopo essere stata sfrattata, viveva in una baracca e richiedeva di poter accedere alle case di Tormarancia «data la necessità di avere una piccola bambina mangiata da tutte sorte di insetti» ${ }^{73}$. Sono molte le donne che, vivendo in condizioni di bisogno, vengono ospitate nella nuova borgata,

\footnotetext{
${ }^{69}$ ASC, Ufficio Assistenza Sociale, Carteggio senza titolario, richieste di ricovero e alloggio, b. 24, f. 1.

${ }^{70}$ Ibidem.

${ }^{71}$ Così venivano definiti i baraccati dal delegato del Governatorato ai servizi assistenziali, Raffaello Ricci nella rivista Capitolium del marzo 1930.

${ }^{72}$ ASC, Ufficio Assistenza Sociale, Carteggio senza titolario, richieste di ricovero e alloggio, b. 24 , f. 1.

${ }^{73}$ Ibidem.
} 
vite al limite che in modo silente cercavano di sopravvivere nella Roma fascista. Da una nota ${ }^{74}$ del custode della borgata Sette Chiese possiamo apprendere la storia di due donne, la moglie e la figlia di tale Francesco De Angelis, alcolista, che non accettava l'amore tra la figlia Virginia ed un ragazzo milite. Infine De Angelis, come si evince dalla lettera della Milizia Volontaria per la Sicurezza Nazionale distaccamento Portuense, Garbatella, Sette Chiese, verrà sfrattato dagli Alberghi Suburbani per morosità e sua moglie e sua figlia furono costrette a vivere all'addiaccio. Saranno proprio i custodi sopracitati che peroreranno la causa di questa disgraziata famiglia richiedendo un alloggio a Tormarancia. Già nel 1930, prima di trovare alloggio la loro situazione era pessima: da una nota della Questura di Roma si viene a conoscenza che tutta la famiglia era stata fermata mentre tentava di costruire una baracca presso Galleria Colonna.

Donne, figlie, ma anche mogli infedeli: questo è il caso di Alessandro Cortonessi, che arriverà a Tormarancia dopo la fine del suo matrimonio. Una memoria depositata all'Ufficio Assistenza Sociale per scongiurare lo sfratto del Cortonesi dalla sua abitazione ci spiega meglio la vicenda:

Non è affatto esatto che il Cortonessi sia stato sfrattato per immoralità dal precedente alloggio delle Case Popolari. E' bensì vero che quando la di lui moglie, Lanciani Clelia, fuggi via con l'amante abbandonandolo con due tenere creature, essendo il Cortonessi costretto a mettersi una donna in casa per la custodia dei suoi bambini, l'Istituto, sobillato da qualche vicina amica della moglie, ottiene alle spalle ed in contumacia del Cortonessi una sentenza di sfratto per il pretesto motivo della non moralità ${ }^{75}$.

Immoralità? Sobillazioni? Vittima della moglie? Poco importa, lo sfratto venne eseguito e successivamente il Cortonessi venne ricollocato proprio nella borgata di Tormarancia. Altra storia quella di Natalina Sacchi, sfrattata perché morosa ma anche perché l'Ufficio d'igiene non permetteva la permanenza della famiglia nei locali: lo stato dei luoghi era tale da non tollerare che ci si potesse vivere. Uno sfratto è quello che pendeva anche su Rosa Ignazio, vedova di guerra e sola con bambini a carico: sia per lei che per la Sacchi le vicissitudini si conclusero con l'arrivo a Tormarancia.

Prima di accedere ad un alloggio tra i giacigli di fortuna che si potevano scegliere c'erano anche i pianerottoli dei palazzi, come per Giovanni Bianchi, anche lui futuro abitante di Tormarancia, che di notte dormiva per le scale di un palazzo in via Porta Angelica, segnalato dalla Questura di Roma in seguito alle lamentele degli inquilini.

Come si è visto, la vita tra le baracche e la strada non era facile, ma neanche nei ricoveri realizzati dal Governatorato le condizioni di vita erano delle migliori. Al 1933 cinque erano i

\footnotetext{
${ }^{74}$ ASC, Ufficio Assistenza Sociale, Carteggio senza titolario, richieste di ricovero e alloggio, b. 23, f. 1.

${ }^{75}$ Ibidem.
} 
ricoveri presenti a Roma: Tuscolano, Sette Chiese, Appio, Casermette e Portuense; in questi potevano essere accolte fino a 417 famiglie $^{76}$, ma in condizioni limite. Le camere singole arrivavano ad ospitare fino a undici persone insieme, con tutti i problemi igienico-sanitari connessi. Nel ricovero di via Tuscolana c'erano stanze senza finestra e cucina che ospitavano fino a sette persone. Ma la situazione del sovraffollamento non era migliore nelle case a Tormarancia: alcune stanze arrivavano ad ospitare anche quindici persone. Esempio ne è l'esperienza di Cesare Fabi, in attesa di un secondo figlio e alloggiato con la famiglia in una camera dove arrivavano ad abitare fino a sette persone.

Per conoscere al meglio gli abitanti della borgata possiamo affidarci nuovamente alle carte dell'Assistenza Sociale, dalle quali si leggono storie come quella di Umberto Tommasini, abitante di Tormarancia, segnalato dai custodi nel marzio 1934 per un fatto spiacevole. Durante un controllo si erano imbattuti in una situazione spinosa: «un bambino veniva chiuso nella sua camera tutto al buio e per mangiare [il padre] ci dava acqua e pane il bambino si lamentava a bassa voce avendo paura del padre che lo malmenasse ancora barbaramente» ${ }^{77}$. Manesco, indolente rispetto alle giustificate lamentele del figlio di soli quattro anni, il Tomassini, a seguito della denuncia dei custodi, venne arrestato e cacciato dalla camera. Un mosaico sociale complesso quello che si venne a creare in questa nuova borgata, popolata da sbaraccati, sfrattati, indigenti.

Gli sfratti però non erano solo il motore che portava i più disperati a Tormarancia, ma avvenivano anche in questa borgata e, da quanto riportano alcuni abitanti in una nota indirizzata direttamente al Duce, sembra che fossero molto frequenti:

\begin{abstract}
Noi, operai, ben anche la disoccupazione abbiamo fatto dei sacrifici per mantenersi la casa, avere una casa, è quello che necessita per noi. L'inverno per noi è stato un grande ostacolo data la scarsità del lavoro, ma ora con la stagione favorevole, considerando la grande ed operosa iniziativa di voi o Duce noi possiamo lavorare, noi lavoreremo perché lo vediamo con i nostri occhi, e allora lavorando possiamo contribuire verso il Governatorato per il pagamento della pigione ${ }^{78}$.
\end{abstract}

Una seconda lettera, sempre del 1934, riporta le parole di un mittente sconosciuto, non iscritto al PNF, ma estimatore del Duce, che, dopo essere stato a Tormarancia e aver conosciuto alcuni abitanti che stavano per essere sfrattati, chiedeva a Mussolini di allungare il tempo concesso agli sfrattati per abbandonare il proprio alloggio, a quel tempo fissato a tre giorni dalla notifica. La lettera si chiudeva con un'esortazione: «Duce! Un po' più di umanità, oltre le strade sontuose.

\footnotetext{
${ }^{76}$ SALVATORI, Paola, Il governatorato di Roma: l'amministrazione della capitale durante il fascismo, Milano, Franco Angeli, 2006, p. 56.

${ }^{77}$ ASC, Ufficio Assistenza Sociale, Carteggio senza titolario, richieste di ricovero e alloggio, b. 33 , f. 1.

${ }^{78}$ ASC, Ufficio Assistenza Sociale, Carteggio con titolario, classe 6, Ricoveri, b. 84, f. 3.
} 
Duce! L'E.V. che proclama di essere col popolo e per il popolo: A noi!»» ${ }^{79}$ Ancora più singolare la firma in calce: «Un benestante che aiuta del proprio questi disgraziati, che con tutta la buona volontà, non trovano lavoro»" ${ }^{80}$.

Tormarancia è anche vita vissuta tutti i giorni, quotidianità. Non era una borgata mite e silenziosa, ed è chiaro anche dalla nota fatta dal custode nell'agosto del 1934 riguardo a balli e canti in alcune case dove gli abitanti invitavano parenti per fare festa anche a tarda notte. Il custode, dopo aver intimato la fine della festa, veniva minacciato, come lui stesso racconta: «L'amante del Carletti, assieme coi parenti invece di eseguire gli ordini da noi dati, si rivolsero con parole triviali e compromettenti, dicendomi persino che se non stavo zitto mi avrebbero fatto la cappotta perché al sottoscritto non lo conoscevano per niente» ${ }^{81}$. I custodi registrarono anche altre liti, tra cui una avvenuta per gelosia tra moglie, marito e la sua presunta amante, un caso più volte segnalato e che generava problemi alla quiete pubblica; oppure gli scontri e le querele per parole infamanti sulla moglie di un uomo o una lite per un bicchiere non riconsegnato. Oggetto di lamentela sono anche i ragazzi della borgata maleducati e protagonisti di atti vandalici.

Dalla documentazione citata emergono storie complesse, capifamiglia in carcere, soldati lontani dalle mogli, donne alcolizzate ${ }^{82}$, altre apostrofate come «equivoche» ${ }^{83}$. Un tessuto sociale che parla e racconta la storia di questo quartiere di Roma, una narrazione polifonica costituita dalla vita dei più bisognosi e dalle vicende di vita quotidiana tra gli sfollati e i baraccati della prima metà del Novecento. La storia del crescere di una comunità va ricostruita dalle sue basi e il fermento a cui assistiamo oggigiorno rispetto allo studio delle borgate romane è lo specchio del tentativo di indagare i fenomeni presenti attualmente a Roma attraverso un'analisi storica di più lungo raggio, volta a costruire le chiavi di lettura adatte a leggere il presente, sempre più complesso. Fenomeni sociali quali l'abbandono scolastico e sacche di microcriminalità hanno radicate ragioni d'essere che, a volte, si intersecano con lo sviluppo degli spazi urbani, con la matrice sociale alla base degli insediamenti, con l'assenza di servizi pubblici e dell'attenzione dovuta da parte delle istituzioni. Un attento studio sociale rispetto al quartiere di Tormarancia potrebbe riconsegnare interessanti spunti per leggere la situazione attuale del quartiere, limbo tra il vicino centro e la periferia sociale. Questo saggio vuole dare un contributo in tal senso, focalizzando l'attenzione sugli abitanti e sulle loro complesse storie di migrazioni, povertà, fatica, di vita quotidiana, dando un ruolo nella storia urbana ai residenti, trovandogli spazio nella narrazione, cercando attraverso le loro vicende di guardare al quartiere di oggi e leggerne aspetti ancora non visti e non approfonditi.

\footnotetext{
${ }^{79}$ Ibidem.

${ }^{80}$ Ibidem.

${ }^{81}$ Ibidem.

${ }^{82}$ ASC, Ufficio Assistenza Sociale, Carteggio con titolario, classe 6, Ricoveri, b. 84, f. 3.

${ }^{83}$ Ibidem.
} 


\section{L'AUTORE}

Flavio CONIA, archivista, ha conseguito la maturità classica e poi la laurea triennale in Storia Moderna e Contemporanea con una tesi in Archivistica Generale e la laurea magistrale in Archivistica e Biblioteconomia con una tesi in Storia degli Archivi, entrambi i corsi di studio svolti presso l'Università di Roma La Sapienza. Nel 2015 entro a far parte del team di ricerca che si occupa del progetto "Rapporto tra scienza, industria e apparato militare nello specchio degli Osservatori Industriali" finanziato da La Sapienza. Borsista presso l'Archivio Centrale dello Stato nel 2017, attualmente dottorando in Scienze documentarie, linguistiche e letterarie, curriculum di Scienze del libro e del documento presso La Sapienza.

URL: < http://www.studistorici.com/progett/autori/\#Conia $>$ 